\title{
The Added Diagnostic Value of Liquid Gastric Emptying Compared with Solid Emptying Alone
}

\author{
Harvey A. Ziessman ${ }^{1}$, Ankit Chander ${ }^{1}$, John O. Clarke ${ }^{2}$, Alison Ramos ${ }^{1}$, and Richard L.Wahl ${ }^{1}$ \\ ${ }^{I}$ Division of Nuclear Medicine, Russell H. Morgan Department of Radiology and Radiological Sciences, Johns Hopkins Medical \\ Institutions, Baltimore, Maryland; and 2 Division of Gastroenterology, Department of Medicine, Johns Hopkins Medical Institutions, \\ Baltimore, Maryland
}

\begin{abstract}
The medical literature states that solid gastric-emptying studies are more sensitive for the detection of gastroparesis than are liquid studies; thus, liquid studies are rarely required. However, we have seen patients with normal solid but delayed liquid emptying. The purpose of this investigation was to determine whether a study of clear liquid gastric empting has added value for the diagnosis of gastroparesis over a study of solid emptying alone. Methods: A total of 101 patients underwent both solid and liquid gastric-emptying studies, acquired sequentially on the same day. A 30-min (1-min frames) liquid study $(300 \mathrm{~mL}$ of water with $7.4 \mathrm{MBq}\left[0.2 \mathrm{mCi}\right.$ ] of ${ }^{111} \mathrm{In}$-diethylenetriaminepentaacetic acid) was followed by a standardized 4-h solid-meal study (a ${ }^{99 m}$ Tcsulfur colloid-labeled egg-substitute sandwich meal). Emptying was quantified as a best-fit exponential emptying rate (T1/2) for liquids and percentage emptying at $4 \mathrm{~h}$ for solid empting. Thirty healthy volunteers underwent a study of clear liquid emptying to establish normal values. The results of the liquid and solid studies were compared. ${ }^{111}$ In liquid downscatter into the subsequent ${ }^{99 m}$ Tc solid meal results was analyzed. Results: The upper range of normal for clear liquid emptying (T1/2) for healthy volunteers was $22 \mathrm{~min}$ (mean \pm 3 SDs) and $19 \mathrm{~min}$ (mean \pm 2 SDs). Of 101 patients, delayed emptying was found in $36 \%$ of liquid and $16 \%$ of solid studies. Of all patients with normal solid emptying, $32 \%$ had delayed liquid emptying. ${ }^{111}$ In downscatter into the $99 \mathrm{~m}$ Tc window was not generally significant. Conclusion: For the detection of gastroparesis, a 30-min study of clear liquid gastric-emptying has considerable added diagnostic value over a study of solid emptying alone.
\end{abstract}

Key Words: gastric emptying; gastroparesis; stomach

J Nucl Med 2009; 50:726-731

DOI: 10.2967/jnumed.108.059790

$\mathbf{T}$ he radionuclide gastric-emptying study has long been the standard clinical diagnostic test for the detection of gastroparesis. Both solid and liquid studies have been used over the years, either as individual or as dual-phase studies,

Received Nov. 3, 2008; revision accepted Jan. 7, 2009.

For correspondence or reprints contact: Harvey A. Ziessman, Johns Hopkins Outpatient Center, 601 N. Caroline St., Suite 3231, Baltimore, MD 21278

E-mail: hziessm1@jhmi.edu

COPYRIGHT $\odot 2009$ by the Society of Nuclear Medicine, Inc. for investigative and clinical purposes. Standard teaching has been that only a solid study is needed for clinical purposes because the liquid study is less sensitive for the detection of gastroparesis, and liquid studies should be reserved for patients who cannot tolerate solids (1-6).

However, we have observed patients who have had delayed liquid but normal solid emptying (7). The primary purpose of this investigation was to determine, in a large patient population, whether liquid gastric emptying provided added diagnostic value over solid emptying alone for the diagnosis of gastroparesis.

We chose to perform the studies on the same day to avoid potential problems (e.g., different fasting conditions, different medications, and possible intercurrent clinical conditions that might affect emptying) that might arise from separate-day studies. Thus, the clear liquid and solid gastricemptying studies were performed sequentially; that is, a 30min water study was performed, followed by the 4-h simplified and standardized solid protocol recommended by recently published consensus recommendations (8). A simultaneous dual-isotope dual-phase study was considered but not used for several reasons. First, the liquid-only study had been our routine liquid methodology, and the cases of discordance that we observed between solid and liquid studies had been noted using the clear liquid-only study. Additionally, there were no published normal values for liquid emptying performed in conjunction with the standardized solid gastric-emptying protocol reported by Tougas et al. (9).

As part of this investigation, we also sought to establish normal values for clear liquid emptying in 30 healthy volunteers. Previously, only a few subjects had been reported. Finally, we analyzed whether ${ }^{111}$ In downscatter from the liquid study into the subsequent solid study ${ }^{99 \mathrm{~m}} \mathrm{Tc}$ window could affect the solid emptying results.

\section{MATERIALS AND METHODS}

\section{Patient Studies}

Between December 2007 and October 2008, sequential liquid and solid radionuclide gastric-emptying studies were performed in 
101 patients ( 24 men, 77 women; age range, 17-77 y; mean \pm SD, $48 \pm 15 \mathrm{y})$. The patients were referred because of symptoms suggestive of gastroparesis. Symptoms included postprandial fullness, epigastric discomfort or pain, nausea, and vomiting. No patients had undergone prior gastric surgery. Seven patients had diabetes mellitus. Patients were taking various medications as ordered by their referring physicians, and the referring physicians decided whether the medications should be discontinued. Patients were instructed not to eat after dinner the night before, to fast overnight, and to have nothing by mouth the morning of the study. The sequential liquid and solid studies were performed in the early morning as the first study of the day. A dual-head large-field-of-view $\gamma$-camera with medium-energy collimators was used for both studies. Patients ingested $300 \mathrm{~mL}$ of water from a commercial water cooler, with 7.4 $\mathrm{MBq}(0.2 \mathrm{mCi})$ of ${ }^{111} \mathrm{In}$-diethylenetriaminepentaacetic acid (DTPA), while lying semiupright $\left(45^{\circ}\right)$ on a hospital gurney. The liquid study was acquired using only 1 of the detector heads positioned in the left anterior oblique projection so that the stomach and upper abdomen were in the field of view and yet the patient could drink the liquid without difficulty. Imaging started immediately after the ingestion of the water. The patients could more easily ingest the liquid in the semiupright position than they could in the supine position, and imaging could be started more promptly after ingestion. Images were acquired as 1 -min frames $\times 30(128 \times 128$ matrix $)$ using a $20 \%$ window around the ${ }^{111}$ In photopeaks (171 and $247 \mathrm{keV}$ ).

Immediately after the 30 -min liquid study was performed, the patient ingested the solid meal. The methodology and meal recommended in a recently published consensus report (8) were closely followed. The meal consisted of a commercially available egg substitute (equivalent to 2 large eggs), mixed with $74 \mathrm{MBq}$ $(2 \mathrm{mCi})$ of ${ }^{99 \mathrm{~m}} \mathrm{Tc}$-sulfur colloid and cooked in a microwave oven for $2 \mathrm{~min}$; 2 slices of toast; strawberry jam; and $120 \mathrm{~mL}$ of water (9). Patients ingested the solid meal within 10-15 min while sitting in an upright position. Patients then lay supine on the imaging table. Simultaneous anterior and posterior images were acquired for $1 \mathrm{~min}$ each $(256 \times 256$ matrix $)$, with a $20 \%$ window centered around the $99 \mathrm{~m}$ Tc photopeak $(140 \mathrm{keV})$. Images were acquired immediately (time 0 ) and at 1, 2, 3, and $4 \mathrm{~h}$ after the meal was ingested.

Both studies were processed on a Xeleris (GE Healthcare) workstation. Regions of interest were drawn around the stomach using a computer, and time-activity curves were generated. For liquid emptying, a half-emptying time (time required for the emptying of half the meal) and a best-fit exponential emptying rate (T1/2) were calculated. Liquid gastric-emptying studies were not attenuation-corrected; prior investigations have shown that correction is not necessary for liquid gastric emptying $(10,11)$. Solid studies were corrected for attenuation and radioactive decay. The geometric mean method (square root of the product of the anterior and posterior counts) was used at each imaging time. The percentage solid gastric emptying was quantified at 1, 2, 3, and $4 \mathrm{~h}$. Normal values for solid emptying were based on the published results of Tougas et al. (9). Data from our study are reported as the percentage emptying rather than as the percentage retention used by Tougas et al. Emptying of less than $90 \%$ at $4 \mathrm{~h}$ was considered delayed. If the patient reached greater than $90 \%$ gastric emptying (the 4-h normal value) at any imaging time, the study was concluded.

\section{Healthy Volunteers}

To establish normal values for clear liquid emptying (water), 30 healthy volunteers were recruited (17 women, 13 men; age range,
24-57 y; mean age $\pm \mathrm{SD}, 41 \pm 12 \mathrm{y})$. The volunteers' body weight ranged from 49.5 to $117 \mathrm{~kg}$ (110-260 lbs) (mean $\pm \mathrm{SD}$, $73.8 \pm 21.15 \mathrm{~kg}[164 \pm 47 \mathrm{lbs}]) ; 15$ volunteers were African American, 12 were Caucasian, and 3 were Asian. The volunteers were judged to be healthy if they had no recent, recurrent, or chronic symptoms or diseases; had not undergone prior abdominal surgery; did not have diabetes mellitus, gastrointestinal symptoms, or chronic illnesses; and were not taking medications (2 subjects were taking a daily low-dose oral contraceptive and several patients were taking over-the-counter vitamins). The study acquisition, processing, and quantification were identical to those described for the patient study, except that $37 \mathrm{MBq}(1 \mathrm{mCi})$ of ${ }^{99 \mathrm{~m}} \mathrm{Tc}-\mathrm{DTPA}$ (rather than $7.4 \mathrm{MBq}[0.2 \mathrm{mCi}]$ of ${ }^{111}$ In-DTPA) in water were used because of the lower radiation dose to the volunteers from the 99m Tc-DTPA.

\section{Investigation of Potential ${ }^{111}$ In Downscatter}

Using our sequential combination liquid-solid gastric-emptying studies, we determined that residual ${ }^{111}$ In-DTPA from the liquid emptying study might still be present in the stomach or cleared into the adjacent small bowel during the ${ }^{99 \mathrm{~m}} \mathrm{Tc}$-sulfur colloid solid emptying study, depending on the degree of gastric emptying. ${ }^{111}$ In downscatters into the ${ }^{99 \mathrm{~m}} \mathrm{Tc}$ window, potentially altering the results of the ${ }^{99 \mathrm{~m}} \mathrm{Tc}$ study. We sought to determine and quantify the effects of ${ }^{111}$ In-DTPA, as observed in real data, and predict when the presence of ${ }^{111}$ In would have the greatest effect.

True solid emptying is calculated by determining the percentage of ${ }^{99 \mathrm{~m}} \mathrm{Tc}$ emptying, not the percentage of measured activity emptying. That is, at time zero $(\mathrm{T}=0 \mathrm{~h})$, immediately after ingestion of the solid meal, ${ }^{99 \mathrm{~m}} \mathrm{Tc}$ activity in the entire abdomen is the measured activity in the entire abdomen minus any residual ${ }^{111} \mathrm{In}$ activity in the abdomen from the prior ${ }^{111} \mathrm{In}$ liquid emptying study. The ${ }^{111} \mathrm{In}$ activity was measured in the ${ }^{99 \mathrm{~m}} \mathrm{Tc}$ window before administration of ${ }^{99 \mathrm{~m}} \mathrm{Tc}$, in both anterior and posterior projections before the calculation of the solid emptying geometric mean. At $4 \mathrm{~h},{ }^{99 \mathrm{~m}} \mathrm{Tc}$ activity in the stomach is the measured activity in the stomach minus the residual ${ }^{111}$ In activity in the stomach. Although the amount of ${ }^{111}$ In in the stomach was not known, it was modeled as either zero or the maximum amount of ${ }^{111}$ In originally in the stomach. At $4 \mathrm{~h}$, the image processing with our software automatically performed decay correction for ${ }^{99 \mathrm{~m}} \mathrm{Tc}$ $(\mathrm{T} 1 / 2=6 \mathrm{~h})$, resulting in an artificial decay correction for ${ }^{111} \mathrm{In}$ $(\mathrm{T} 1 / 2=67 \mathrm{~h})$ activity scattered into the ${ }^{99 \mathrm{~m}} \mathrm{Tc}$ window. This artificial increase was rectified by the following equation: ${ }^{99 \mathrm{~m}} \mathrm{Tc}$ in the stomach at $4 \mathrm{~h}=$ measured activity in the stomach minus ${ }^{111} \mathrm{In}$ activity in the stomach at $4 \mathrm{~h}$, where ${ }^{111}$ In activity in the stomach at $4 \mathrm{~h}$ can be modeled as zero or as no emptying $\left({ }^{111} \mathrm{In}\right.$ in the stomach at $0 \mathrm{~h}) \times 1.5\left(1.5\right.$ is the ${ }^{99 \mathrm{~m}} \mathrm{Tc}$ decay data-correction factor at $4 \mathrm{~h}$ ).

\section{RESULTS}

Data are mean $\pm \mathrm{SD}$.

\section{Healthy Volunteers}

For the 30 healthy subjects, the liquid water half-emptying time ranged from 6 to $20 \mathrm{~min}(14.3 \pm 4.1 \mathrm{~min})$. The T1/2 exponential-fit clearance rate ranged from 7 to $19 \mathrm{~min}$ (12.4 $\pm 3.1 \mathrm{~min})$. All healthy subjects had an exponential emptying pattern. A delay before emptying began, ranging from 1 to $10 \mathrm{~min}(1.9 \pm 2.6 \mathrm{~min})$, was seen in 12 of 30 
subjects. Because of the more variable and longer halfemptying time (compared with T1/2 exponential-fit clearance rate) due to this delay, the exponential-fit value was used to determine normal. For the mean plus 2 SDs, the upper range of normal T1/2 was 18.6 , or less than $19 \mathrm{~min}$; for the mean plus $3 \mathrm{SDs}$, the upper range of normal was $21.8 \mathrm{~min}$, or less than $22 \mathrm{~min}$.

Of 7 diabetic patients in this study, 4 had normal solid and liquid emptying, 2 had delayed solid and liquid emptying, and 1 had normal solid but delayed liquid emptying. The serum blood glucose was available for 1 patient with normal solid and liquid emptying $(185 \mathrm{dL} / \mathrm{mL})$ and for 1 patient with delayed solid and liquid emptying (145 dL/mL).

\section{Patient Studies}

Figures $1 \mathrm{~A}$ and $1 \mathrm{~B}$ show the distribution of normal and abnormal liquid and solid studies based on normal values of the mean plus 2 and 3 SDs. Of the 101 patient studies, solid emptying was delayed in 16. Liquid emptying was delayed in 39 ( 2 SDs) and 36 (3 SDs), respectively. Thus, liquid emptying was delayed more than twice as frequently as was solid emptying. Both solid and liquid emptying studies were delayed in 12 patients, and solid emptying was delayed but liquid was normal in 4 patients. A total of 27 (2 SDs) and 24 (3 SDs) had delayed liquid but normal solid emptying.

\begin{tabular}{|c|c|c|c|c|}
\hline A & & LIC & UID & \\
\hline & & Normal & Delayed & Total \\
\hline 으 & Normal & 58 & 27 & 85 \\
\hline 心 & Delayed & 4 & 12 & 16 \\
\hline & Total & 62 & 39 & 101 \\
\hline B & & & UID & \\
\hline & & Normal & Delayed & Total \\
\hline 으 & Normal & 61 & 24 & 85 \\
\hline ๘ & Delayed & 4 & 12 & 16 \\
\hline & Total & 65 & 36 & 101 \\
\hline
\end{tabular}

FIGURE 1. (A) Matrix for 101 patient studies with normal and delayed gastric emptying using normal to be 2 SDs around mean $(<19 \mathrm{~min})$ of 30 healthy subjects. (B) Matrix for 101 patient studies with normal and delayed gastric emptying using normal to be 3 SDs around mean ( $<22 \mathrm{~min})$ of 30 healthy subjects.
Of 85 patients with normal solid emptying, 27 (2 SDs; $32 \%$ ) and 24 (3SDs; 28\%) had delayed liquid emptying.

Not all patients ingested the entire solid meal. Of 101 patients, 13 ingested only $50 \%-90 \%$ of the meal. Five of the 13 patients had prolonged liquid emptying with an exponential T1/2 of 28, 35, 40, 41, and $284 \mathrm{~min}$. All had normal solid emptying values; however, because they did not ingest the entire meal, the normal values may not apply.

Patients with a normal T1/2 for liquid emptying had an exponential clearance pattern in 61 of 65 studies $(94 \%)$. Of the 36 patients with a delayed T1/2 (mean \pm 3 SDs), $56 \%$ had an exponential pattern of emptying; $44 \%$ did not. None of the patients with a T1/2 greater than $42 \mathrm{~min}$ had exponential clearance. A delay before emptying began was seen in 41 of 65 patients $(63 \%)$ with a normal T1/2 $(2.7 \pm$ $3.4 \mathrm{~min})$ and in 29 of $36(81 \%)$ of patients with an abnormally prolonged $\mathrm{T} 1 / 2(8.9 \pm 9.5 \mathrm{~min})$. The distribution of delayed T1/2 liquid emptying was 22-24 min (5 patients), 25-30 $\min$ (11 patients), 31-58 $\min$ (12 patients), and 122-526 $\min$ (8 patients).

\section{Downscatter Analysis}

A sample of 20 patient studies was analyzed for the possibility of downscatter from the ${ }^{111} \mathrm{In}$ into the ${ }^{99 \mathrm{~m}} \mathrm{Tc}$ window. If it is assumed that any ${ }^{111} \mathrm{In}$ remaining in the stomach at time zero for the solid study was still present at $4 \mathrm{~h}$, true percentage solid emptying differed from measured percentage solid emptying by $0.15(8.43 \%)$ (Figs. 2A and 2B). If ${ }^{111}$ In was assumed to remain in the stomach at $4 \mathrm{~h}$, $45 \%$ of true percentage solid emptying was less than measured, versus $95 \%$ if no ${ }^{111}$ In was assumed to remain in the stomach. These data suggest that, if all or most of the ${ }^{111} \mathrm{In}$ is in the small bowel at $4 \mathrm{~h}$, true percentage solid emptying tends to be less than measured percentage emptying, the error varies with the magnitude of percentage emptying, and the error is usually small.

The variables of relative total ${ }^{99 \mathrm{~m}} \mathrm{Tc}$ and ${ }^{111} \mathrm{In}$ counts, relative amounts of ${ }^{111} \mathrm{In}$ in the stomach and small bowel at 0 and $4 \mathrm{~h}$, and measured percentage solid emptying were modeled to MATLAB (student version; The MathWorks) to predict when the ${ }^{111}$ In could produce the greatest deviation in percentage solid emptying. The results of the model followed the previously mentioned conclusions made from data. If no ${ }^{111} \mathrm{In}$ is assumed in the stomach at $4 \mathrm{~h}$, the maximum possible error varies linearly with the relative amounts of ${ }^{111} \mathrm{In}$ and ${ }^{99 \mathrm{~m}} \mathrm{Tc}$, with greater error when a greater amount of ${ }^{111}$ In is present.

\section{DISCUSSION}

From a functional standpoint, the stomach has 2 compartments: the proximal stomach, or fundus, and the distal stomach, or antrum $(3,12)$. After ingestion of a meal, the fundus relaxes and accommodates the meal volume; the smooth muscle of the fundus contracts in a tonic manner, producing a pressure gradient between the fundus and the pylorus, which is responsible for liquid emptying. In contrast, 

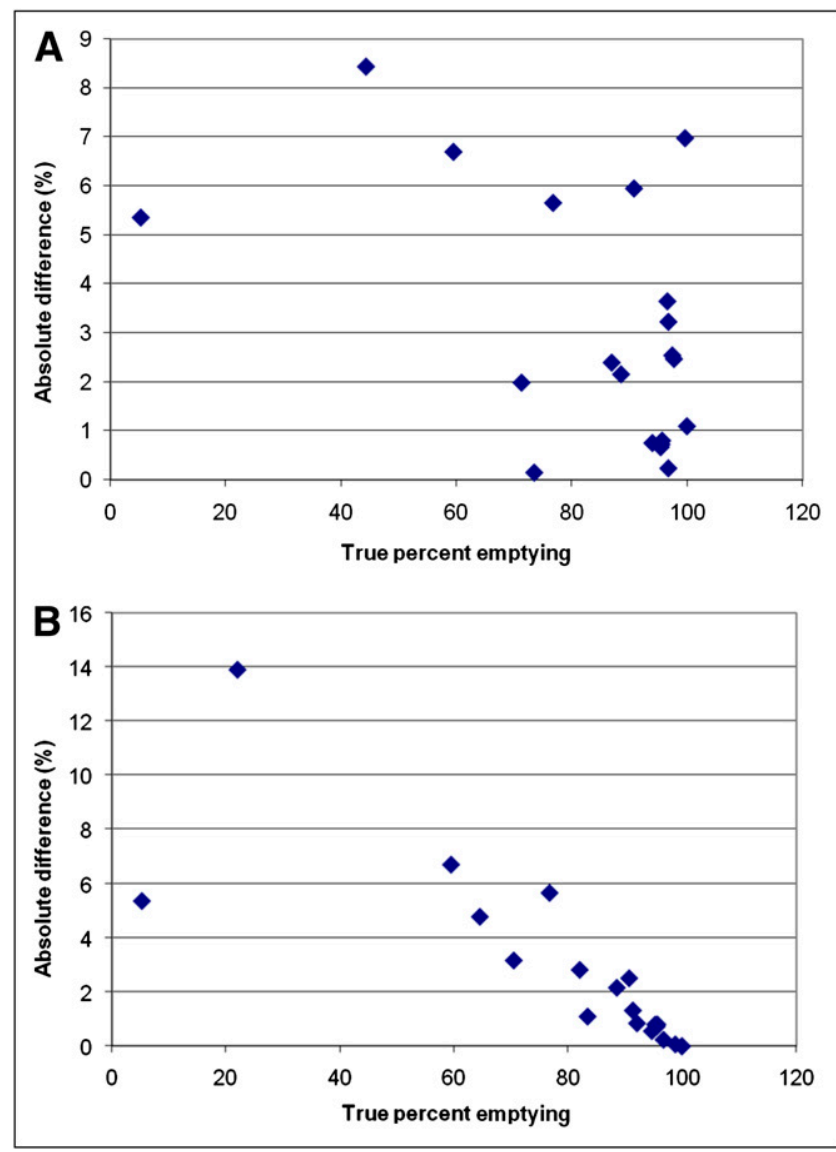

FIGURE 2. (A) Absolute difference from measured percentage solid gastric emptying vs. true percentage emptying at $4 \mathrm{~h}$, assuming maximum ${ }^{111} \mathrm{In}$ in stomach at $4 \mathrm{~h}$. (B) $\mathrm{Ab}-$ solute difference from measured percentage solid gastric emptying vs. true percentage emptying, assuming no ${ }^{111} \mathrm{In}$ in stomach at $4 \mathrm{~h}$.

the antrum produces strong phasic muscular contractions that grind up solid food into small particles that can pass through the pylorus; the antrum is responsible for solid emptying.

Standard teaching has long been that solid radionuclide gastric emptying is more sensitive for the detection of gastroparesis than is liquid emptying. This is stated in gastrointestinal (4-6) and nuclear medicine review articles (13-15), textbooks $(1,16)$, and Society of Nuclear Medicine Procedure Guidelines (17). This consensus seems to have been based on observations made from dual-phase, dualisotope solid-liquid gastric-emptying studies (18-23). As a result, only solid gastric-emptying studies are recommended for clinical purposes; liquid studies are needed only in special situations, for example, for patients who cannot tolerate solid meals, patients who are postoperative, or patients with suspected dumping syndrome $(4,5,16)$.

A potential problem with accepting the results from dualphase studies is that the emptying of the liquid phase is directly affected by the solid-phase meal. For example, the addition of calories in the form of $10 \%$ dextrose to a clear liquid meal slows the emptying rate, although the exponential pattern of emptying is maintained. However, with increasing caloric content (e.g., 25\% dextrose) the emptying rate is slowed further and the pattern of clearance changes, from exponential to linear, similar to that seen in a solid study (20). Thus, the solid phase has a definite effect on the rate of liquid emptying, which may explain the discrepancy between our results and prior reports derived from dual-phase studies.

The Tougas et al. (9) standardized and simplified solidmeal protocol has been recommended in a joint report of the Society of Nuclear Medicine and the American Neurogastroenterology and Motility Society (8). One criticism of this method is that infrequent imaging does not allow for the analysis of the pattern of gastric contractility. The present investigation is the second time that we have integrated a research study and the 4-h Tougas protocol (24). In the prior study, we investigated whether the lag phase could predict delayed emptying. We acquired images every 10 min during the first hour of the 4-h study. The lag phase did not prove to be a predictor of overall emptying (24). However, in that same study, we found that a solid study length of $4 \mathrm{~h}$, compared with the more traditional 2-h study, increased the number of patients diagnosed with gastroparesis by $32 \%$. In the present investigation, we have integrated a 30-min clear liquid study with the Tougas protocol and have found that the clear liquid study detected gastroparesis in approximately $30 \%$ of patients with normal solid empting studies. The diagnosis of gastroparesis increased significantly, from $16 \%$ of patients for the solid-only 4 -h study to an additional $28 \%-32 \%$ of patients who had a normal solid study but abnormal liquid emptying. Thus, the addition of the liquid study to the solid study has considerable added diagnostic value.

Past investigations have found a poor correlation between patient symptomatology and the results of gastric-emptying studies. Abnormal emptying has been found in a relatively low percentage of patients with symptoms suggestive of gastroparesis, who have a clinical diagnosis of nonulcer dyspepsia, idiopathic gastroparesis, and diabetes mellitus $(5,25-27)$. One important reason may be that we have been studying only antral contraction and not fundal contraction.

Previously published data on normal values for clear liquid gastric emptying are limited. In 1974, Chaudhuri reported on noncaloric liquid (saline) gastric emptying in 8 healthy subjects (28). Each subject underwent 3 studies, and the study was shown to have good reproducibility. A normal T1/2 value based on these 24 total studies $(8 \times 3)$ was 6-18 min (mean $\pm \mathrm{SD}, 12 \pm 3 \mathrm{~min}$ ). Two other reports also used a saline clear liquid meal. In 1 study with 7 healthy subjects, the upper level of normal values was less than $20 \mathrm{~min}$ (29), and in another report with an uncertain number of subjects, the upper level was less than $25 \mathrm{~min}$ (30). Our investigation of 30 healthy subjects found normal emptying to be less than 19 min (mean \pm 2 SDs) and less than 22 min (mean \pm 3 SDs). The rate of liquid emptying is, to some extent, position-dependent; the rate is slower with the patient in a supine rather than sitting position (29). 
Unlike solids, clear liquids have been reported to empty in a monoexponential pattern, with no delay before emptying begins (31). However, our study has shown a delay of $1-10 \mathrm{~min}(1.9 \pm 2.6 \mathrm{~min})$ in 13 of 30 healthy subjects. All healthy subjects in our study had an exponential pattern of emptying. In our study, the patients with a moderate delay in liquid emptying maintained an exponential emptying pattern; however, those with more delayed emptying often did not empty exponentially.

There has long been concern about ${ }^{111}$ In downscatter into the ${ }^{99 \mathrm{~m} T c}$ window in dual-isotope studies. Some investigations have used scatter-correction methods to minimize this problem (20); other studies, however, have shown that, if the administered dose of ${ }^{99 \mathrm{~m}} \mathrm{Tc}$ is at least $5-6$ times that of ${ }^{111} \mathrm{In}$, dose downscatter is not a significant problem $(32,33)$. With the sequential methodology described in this article, there was a concern that residual liquid ${ }^{111}$ In might affect the results of the ${ }^{99 \mathrm{~m}} \mathrm{Tc}$ solid study. Using a 10:1 administered dose ratio of ${ }^{99 \mathrm{~m}} \mathrm{Tc}$ to ${ }^{111} \mathrm{In} \mathrm{keV}$ in our study, our analysis showed that the problem of residual liquid is generally not significant using the methodology described.

There have been anecdotal reports of patients who had delayed liquid but normal solid emptying (34). A single published investigation of 85 patients with diabetes reported delayed liquid but normal solid emptying in $24 \%$ of patients (35). This finding was said by the authors to be specific to diabetic patients, and this observation has not been followed up. Our study clearly demonstrates that abnormal liquid but normal solid gastric emptying is not an uncommon finding in a general referral population of patients with symptoms suggestive of gastroparesis. Only 7 of our patients were diabetic but none had delayed liquid/normal solid emptying.

Patients with delayed liquid emptying but normal solid emptying likely have dysfunction of the gastric fundus. Further investigation is needed to determine whether this hypothesis is correct. Several techniques (e.g., barostatic manometry, ultrasonography, MRI, and SPECT) have been described that might help evaluate gastric fundal function (36). If this hypothesis is true, this might lead to new methods of therapy that are more physiologic and might allow for individualized therapy of gastroparesis, depending on whether the patients have antral or fundal dysfunction or both.

\section{CONCLUSION}

We defined the reference range for clear liquid gastric emptying. In a sequential 2-phase protocol, we found that a 30-min clear liquid gastric-emptying study performed immediately before a 4-h standardized solid emptying study is often abnormal when the solid study is normal, and the gastric-emptying study added considerable diagnostic value for the detection of gastroparesis over solid emptying alone.

\section{REFERENCES}

1. Parkman HP, Fisher RS. Disorders of gastric emptying. In: Yamada T, Alpers DH, Kaplowitz N, Laine L, Owyang C, Powell DW, eds. Textbook of Gastroenterology. 4th ed. Philadelphia, PA: Lippincott Williams \& Wilkins; 2003:1292-1310.
2. Lin HC, Prather C, Fisher RS, et al. AMS Task Force Committee on gastrointestinal transit: measurement of gastrointestinal transit. Dig Dis Sci. 2005;50: 989-1004.

3. Hasler WL. Disorders of gastric emptying. In: Yamada T, Alpers DH, Kaplowitz N, Laine L, Owyang C, Powell DW, eds. Textbook of Gastroenterology. 3rd ed. Philadelphia, PA: Lippincott Williams \& Wilkins; 1999:1341-1369.

4. Camilleri M, Hasler WL, Parkman HP, Quigley EM, Soffer E. Measurement of gastrointestinal motility in the GI laboratory. Gastroenterology. 1998;115:747-762.

5. Parkman HP, Hasler WL, Fisher RS. American Gastroenterological Association technical review on the diagnosis and treatment of gastroparesis. Prepared by the American Gastroenterological Association Clinical Practice Committee. Gastroenterology. 2004;127:1592-1622.

6. Hornbuckle K, Barnett JL. The diagnosis and work-up of the patient with gastroparesis. J Clin Gastroenterol. 2000;30:117-124.

7. Ziessman HA, Okolo P, Mullin G, Chander A. The added diagnostic value of liquid gastric emptying when solid emptying is normal [abstract]. $\mathrm{J} \mathrm{Nucl} \mathrm{Med}$. 2008;49(suppl 1):39P.

8. Abell TL, Camilleri M, Donohoe K, et al. Consensus recommendations for gastric emptying scintigraphy: a joint report of the Society of Nuclear Medicine and the American Neurogastroenterology and Motility Society. J Nucl Med Technol. 2008;36:44-54.

9. Tougas G, Eaker EY, Abell TL, et al. Assessment of gastric emptying using a low fat meal: establishment of international control values. Am J Gastroenterol. 2000;95:1456-1462.

10. Collins PJ, Horowitz M, Cook DJ, et al. Gastric emptying in normal subjects: a reproducible technique using a single scintillation camera and computer system. Gut. 1983;24:1117-1125.

11. Christian PE, Moore JG, Sorenson JA, et al. Effects of meal size and correction technique on gastric emptying time: studies with two tracers and opposed detectors. J Nucl Med. 1980;21:883-885.

12. Kelly KA. Gastric emptying of liquids and solids: roles of proximal and distal stomach. Am J Physiol. 1980;239:G71-G76.

13. Maurer AH, Parkman HP. Update on gastrointestinal scintigraphy. Semin Nucl Med. 2006;36:110-118.

14. Datz FL. Considerations for accurately measuring gastric emptying. J Nucl Med. 1991;32:881-884.

15. Mariani G, Boni G, Barreca M, et al. Radionuclide gastroesophageal motor studies. J Nucl Med. 2004;45:1004-1028.

16. Ziessman HA, O'Malley J, Thrall JH. Gastrointestinal system. In: Ziessman HA, O'Malley J, Thrall JH, eds. Nuclear Medicine: The Requisites. Philadelphia, PA: Mosby; 2006.

17. Donohoe KJ, Maurer AH, Ziessman HA, et al. Procedure guideline for gastric emptying and motility. J Nucl Med. 1999;40:1236-1239.

18. Couturier O, Bodet-Milin C, Querellou S, et al. Gastric scintigraphy with a liquid-solid radiolabelled meal: performances of solid and liquid parameters. Nucl Med Commun. 2004;25:1143-1150.

19. Heading RC, Tothill P, McLoughlin GP, Shearman DJC. Gastric emptying role measurement in man: a double isotope scanning technique for simultaneous study of liquid and solid components of a meal. Gastroenterology. 1976;71:45-50.

20. Fisher RS, Malmud LS, Bandid P, et al. Gastric empting of a physiologic mixed solid-liquid meal. Clin Nucl Med. 1982;7:215-221.

21. Collins PJ, Houghton LA, Read NW, et al. Role of the proximal and distal stomach in mixed solid and liquid meal emptying. Gut. 1991;32:615-619.

22. Wright RA, Thompson D, Syed I. Simultaneous markers for fluid and solid gastric emptying: new variations on an old theme-concise communication. J Nucl Med. 1981;22:772-776.

23. Moore JG, Christian PE, Coleman RE. Gastric empting of varying meal weight and composition in man. Dig Dis Sci. 1981;26:16-22.

24. Ziessman HA, Bonta DV, Goetze S, Ravich WJ. Experience with a simplified, standardized 4-hour gastric-emptying protocol. J Nucl Med. 2007;48:568-572.

25. Horowitz M, Harding PE, Maddox AF, et al. Gastric and oesophageal emptying in patients with type 2 (non-insulin-dependent) diabetes mellitus. Diabetologia. 1989;32:151-159.

26. Talley NJ, Shuter B, McCrudden G, et al. Lack of association between gastric emptying of solids and symptoms in nonulcer dyspepsia. J Clin Gastroenterol. 1989;11:625-630.

27. Koch KL, Stern RM, Stewart WR, Vasey MW. Gastric emptying and gastric myoelectrical activity in patients with diabetic gastroparesis: effect of long-term domperidone treatment. Am J Gastroenterol. 1989;84:1069-1075.

28. Chaudhuri TK. Use of ${ }^{99} \mathrm{Tc}-\mathrm{DTPA}$ for measuring gastric empting time. $\mathrm{J} \mathrm{Nucl}$ Med. 1974;15:391-395.

29. Anvari M, Horowitz M, Fraser R, et al. Effects of posture on gastric emptying of nonnutrient liquids and antropyloroduodenal motility. Am J Physiol Gastrointest Liver Physiol. 1995;31:G868-G871. 
30. Tamas S, Dumitrascu DL, Andreica V, Cotul S. Gastric sequential scintigraphy: methodology with liquid isotonic meal. Physiologie. 1988;25:47-51.

31. Hunt JN, Spurrell WR. The pattern of emptying of the human stomach. J Physiol. 1951;113:157-168.

32. Christian PE, Datz FL, Sorenson JA, et al. Technical factors in gastric emptying studies. J Nucl Med. 1983;24:264-267.

33. Ziessman HA, Fahey FH, Collen MJ. Biphasic solid and liquid gastric empting in normal controls and diabetics using continuous acquisition in LAO view. Dig Dis Sci. 1992;37:744-750.
34. Lin HC, Hasler WJ. Disorders of gastric emptying. In: Yamada T, Alpers DH, Kaplowitz N, Laine L, Owyang C, Powell DW, eds. Textbook of Gastroenterology. 2nd ed. Philadelphia, PA: JB Lippincott Company, 1995:1318-1346.

35. Horowitz M, Maddox AF, Wishart JM, Harding PE, Chatterton BE, Shearman DJ. Relationships between oesophageal transit and solid and liquid gastric emptying in diabetes mellitus. Eur J Nucl Med. 1990;18:229-234.

36. Kim D-Y, Myung S-J, Camilleri M. Novel testing of human gastric motor and sensory functions: rationale, methods, and potential applications in clinical practice. Am J Gastroenterol. 2000;95:3365-3373. 